\title{
The next step towards anticancer microbiota therapeutics
}

\section{Ef \\ a consortium \\ of 11 bacteria \\ from the \\ human gut \\ microbiota that \\ can increase \\ the number of \\ crucial $\mathrm{CD}^{+}$ \\ $T$ cells and the \\ efficacy of \\ immune \\ checkpoint \\ inhibitors}

The gut microbiota can influence the response to and outcome of anticancer therapies, including immune checkpoint inhibitors targeting programmed cell death protein 1 (PD1) or cytotoxic T lymphocyte antigen 4 (CTLA4). One barrier to exploiting this link and developing microbiota-based therapeutics that improve cancer treatment is the difficulty in identifying the responsible microbial species, which is a prerequisite for then elucidating their characteristics, effects, safety, etc. Kenya Honda and colleagues have now isolated a consortium of 11 bacteria from the human gut microbiota that can increase the number of crucial $\mathrm{CD}^{+}$ $\mathrm{T}$ cells and the efficacy of immune checkpoint inhibitors.

The human gut microbiota is highly diverse and complex and has manifold effects on the host that might be relevant for anticancer therapies. In this study, Honda and colleagues focused on the induction of $\mathrm{CD}^{+} \mathrm{T}$ cells that produce the effector cytokine interferon- $\gamma$ $(\mathrm{IFN} \gamma)$. IFN $\gamma^{+} \mathrm{CD} 8^{+} \mathrm{T}$ cells are crucial not only in the immune response against cancer but also

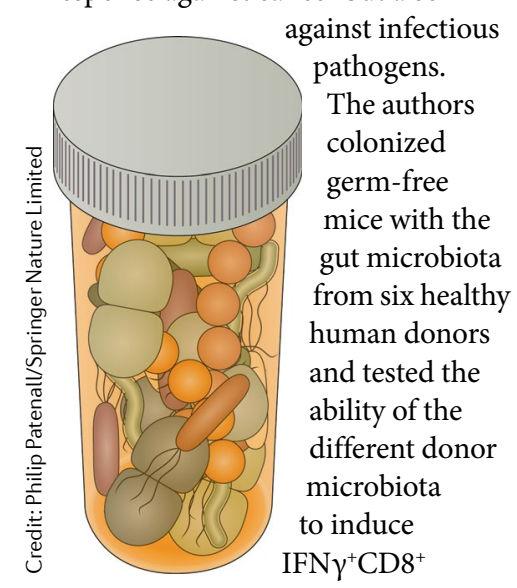

$\mathrm{T}$ cells in the colon. Induction varied substantially between donors, so they then selected the microbiota in the mouse with the strongest induction for further study.

To identify the specific bacteria responsible for induction, they performed secondary transplants and treated the recipient mice with different antibiotics. Metronidazole, streptomycin and tylosin reduced induction, whereas ampicillin increased induction. Interestingly, the microbiota pretreated with chloroform, which kills nonspore-forming bacteria, abrogated induction. Then, they isolated 26 unique bacterial strains from the ampicillin-treated mice, although 5 were excluded because they were found in mice inoculated with the chloroform-treated microbiota. The abundance of 11 of the remaining 21 strains positively correlated with IFN $\gamma^{+} \mathrm{CD}^{+} \mathrm{T}$ cell levels, whereas the other 10 showed no association. Indeed, a consortium of the 11 positively correlated strains stably induced IFN $\gamma^{+} \mathrm{CD}^{+} \mathrm{T}$ cells locally and systemically, without any sign of colon inflammation in germ-free mice, whereas the other 10 strains had no effect.

All 11 strains are rare members of the human microbiota, including several Bacteroides spp. and Parabacteroides spp., Eubacterium limosum,

Ruminococcaceae bacterium cv2, Phascolarctobacterium faecium and Fusobacterium ulcerans. Importantly, their genomes contained no obvious toxins and virulence factors and no multidrug resistance markers.

Fluorescence in situ hybridization showed that the strains colonize the mucus layer right above the colonic epithelium, where they induce the production of chemokines and promote the proliferation and accumulation of $\mathrm{CD} 8^{+} \mathrm{T}$ cells. Mutant mice with defective or absent dendritic cells showed no induction, whereas innate signalling pathways had no involvement. By contrast, the major histocompatibility complex Ia, which is involved in antigen presentation to $\mathrm{CD}^{+} \mathrm{T}$ cells, was necessary for induction.

Finally, Honda and colleagues confirmed the therapeutic potential of the 11-strain consortium in mice infected with Listeria monocytogenes and in mice with tumour xenografts. Interestingly, the 11-strain consortium not only improved the efficacy of anti-PD1 and anti-CTLA4 therapy but also boosted spontaneous antitumour immunity in the absence of immune checkpoint inhibition in multiple cancer models. Of note, the 11-strain consortium had a beneficial anticancer effect not only in germ-free mice but also in specific pathogen-free mice and in mice colonized with human gut microbiota from a donor that had shown low IFN $\gamma^{+} \mathrm{CD}^{+} \mathrm{T}$ cell levels in the initial experiments.

In summary, Honda and colleagues have isolated a consortium of 11 human gut commensals that can stimulate anticancer and antibacterial $\mathrm{CD}^{+} \mathrm{T}$ cell responses, which makes these strains promising candidates for microbiota-based therapeutics.

Ursula Hofer

ORIGINAL ARTICLE Tanoue, T. \& Morita, S. et al. A defined commensal consortium elicits CD8 T cells and anti-cancer immunity. Nature https://doi.org/10.1038/s41586-019-0878-z (2019) FURTHER READING Zitvogel, L. et al. Anticancer effects of the microbiome and its products. Nat. Rev. Microbiol. 15, 465-478 (2017) 